\title{
Excitation of Kaluza-Klein gravitational mode
}

\author{
Kunihito Uzawa \\ Graduate School of Science and Technology, Chiba University \\ Chiba 263-8522, Japan \\ Yoshiyuki Morisawa \\ Yukawa Institute for Theoretical Physics, Kyoto University \\ Kyoto 606-8502, Japan \\ Shinji Mukohyama \\ Department of Physics and Astronomy, University of Victoria \\ Victoria BC, Canada V8W 3P6 \\ Canadian Institute for Theoretical Astrophysics, University of Toronto \\ Toronto ON, Canada M5S $3 H 8$
}

(August 29, 2018)

\begin{abstract}
We investigate excitation of Kaluza-Klein modes due to the parametric resonance caused by oscillation of radius of compactification. We consider a gravitational perturbation around a $D$ dimensional spacetime, which we compactify on a $(D-4)$-sphere to obtain a 4-dimensional theory. The perturbation includes the so-called Kaluza-Klein modes, which are massive in 4-dimension, as well as zero modes, which is massless in 4-dimension. These modes appear as scalar, vector and second-rank symmetric tensor fields in the 4-dimensional theory. Since Kaluza-Klein modes are troublesome in cosmology, quanta of these Kaluza-Klein modes should not be excited abundantly. However, if radius of compactification oscillates, then masses of Kaluza-Klein modes also oscillate and, thus, parametric resonance of Kaluza-Klein modes may occur to excite their quanta. In this paper we consider part of Kaluza-Klein modes which correspond to massive scalar fields in 4-dimension and investigate whether quanta of these modes are excited or not in the so called narrow resonance regime of the parametric resonance. We conclude that at least in the narrow resonance regime quanta of these modes are not excited so catastrophically.
\end{abstract}

\section{INTRODUCTION}

When we consider unified theories, spacetime may have more than four dimension. For example, the critical dimension of the superstring theories [1] is equal to ten, and the low-energy effective theory of M-theory [2] is the eleven-dimensional supergravity. On the other hand, we recognize only four dimensional spacetime. Therefore, in order to describe our universe we have to adopt some mechanism of dimensional reduction. Compactification of extra dimensions on some compact manifold (the Kaluza-Klein prescription [3]) has been conventionally adopted, while an alternative prescription was recently proposed by Randall and Sundrum [ $\mid$. Since the Randall-Sundrum prescription cannot be applied directly to more than 5-dimension, in this paper we consider the Kaluza-Klein prescription.

However, there are too many ways of compactification to specify a 4-dimensional theory from the higher dimensional unified theories. As for the superstring theory, although it is usually compactified on a Calabi-Yau manifold, there are so many Calabi-Yau manifolds.

Fortunately, in some cases it may be possible to judge which way of compactification is acceptable since different compactifications may give different 4-dimensional theories and, thus, different cosmologies. In particular, properties, eg. mass spectrum, of the so-called Kaluza-Klein modes reflect the manifold on which spacetime is compactified. Thus, it may be effective to investigate effects of Kaluza-Klein modes on the 4-dimensional cosmology.

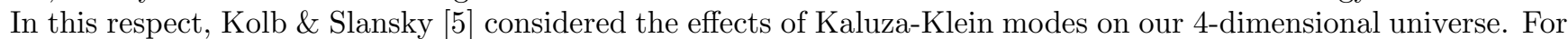
simplicity, they considered a scalar field in 5-dimensional spacetime of the form $M^{4} \times S^{1}$, where $M^{4}$ is a 4-dimensional FRW universe and $S^{1}$ is a circle. They investigated Kaluza-Klein modes of the scalar field and showed that the KaluzaKlein modes are dangerous in cosmology. To be precise, they showed that, if energy density of a Kaluza-Klein mode is not negligible compared with radiation density at an early epoch, then the Kaluza-Klein mode eventually dominate the universe. Thus, quanta of Kaluza-Klein modes should not be excited abundantly. It is expected that this result 
holds in quite general situation since their arguments are based on the momentum conservation along the compact direction. Thus, quanta of Kaluza-Klein modes of any fields should not be excited abundantly.

However, there is a possibility that quanta of Kaluza-Klein modes may be excited. First, the radius of compactification appears as a scalar field in 4-dimension called a radion [6]. Hence, if the radion potential has a local minimum then it can oscillate around the local minimum. The oscillation of the radion leads to oscillation of masses of KaluzaKlein modes since the masses are proportional to the inverse of the compactification radius. On the other hand, it is well-known that oscillation of mass leads to the so-called parametric resonance phenomenon 88.9]. Hence, it is expected that, if the radion potential has a local minimum then quanta of Kaluza-Klein modes may be excited by parametric resonance [10].

Therefore, it is possible to obtain some knowledge about a way of compactification by investigating whether quanta of Kaluza-Klein modes are excited by the parametric resonance due to the radion oscillation. If the quanta are produced abundantly then the way of compactification should be rejected. In Ref. [10, the parametric resonance of Kaluza-Klein modes of a scalar field was investigated in the regime where the resonance band is narrow (the narrow resonance regime). It was shown that, for the case of compactification by a $d$-dimensional sphere, quanta of the Kaluza-Klein modes are not overproduced.

However, since there should be many other fields (eg. gravitational fields, anti-symmetric fields, spinor fields, and so on), the investigation of only a scalar field is not enough: in principle, we have to investigate Kaluza-Klein modes of all fields in the theory. In this paper, we investigate a gravitational field.

This paper is organized as follows. In section [I] we describe our model and derive the action for our system. In section III we investigate whether catastrophic creation of quanta of the Kaluza-Klein modes occur or not. In section IV we summarize this paper.

\section{MODEL CONSTRUCTION}

In this section we derive an effective action for Kaluza-Klein gravitational modes as well as for background fields. In subsection II A we describe our model and basic assumptions verbally. In subsection II B we calculate the tree action of our system from the Einstein-Hilbert action. In subsection II we stabilize the radion potential by the so-called Casimir effect to obtain the effective action for our system.

\section{A. Basic assumptions}

As already explained in Sec. If, our purpose in this paper is to judge whether a particular model of dimensional reduction is acceptable or not by investigating parametric excitation of Kaluza-Klein modes. For this purpose, we have to specify higher dimensional theory in $D$-dimension somewhat. Moreover, in order to obtain the 4-dimensional effective theory from the $D$-dimensional theory, we have to specify a particular model of dimensional reduction. Namely, we consider the following setting.

(a) Let us consider a $D$-dimensional theory which includes gravity, $N_{s}$ scalar fields, $N_{d}$ Dirac fields, and other fields. The gravity, scalar fields and spinor fields are assumed to be described by the Einstein-Hilbert action with a cosmological constant, the Klein-Gordon action and the Dirac action, respectively. There may be interaction among these fields.

(b) We adopt the conventional Kaluza-Klein description of compactification: we compactify the $D$-dimensional spacetime on a $d$-dimensional compact manifold $(d=D-4)$ to obtain the 4-dimensional spacetime. As the compact manifold we take a $d$-dimensional sphere, which we shall denote by $S^{d}$.

(c) We consider the so-called Casimir effect to stabilize a radion potential, where the radion is a 4-dimensional scalar field corresponding to compactification radius [6]. In order to stabilize the radion potential by the Casimir effect, as shown in subsection IIC, we have to assume that $d \geq 2$.

(d) We also consider perturbations of $D$-dimensional fields around the background given by the Kaluza-Klein prescription. These perturbations are described as 4-dimensional massive fields called Kaluza-Klein modes.

For this situation, in order to make calculations possible, we assume the following assumptions. (See the beginning of section III for further assumptions.)

(i) The number $N_{s}$ (or $N_{d}$ ) of scalar fields (or Dirac fields) is sufficiently large compared with the number of other fields included in the $D$-dimensional theory. 
(ii) The cosmological constant induced in 4-dimension is zero.

(iii) Terms in the 4-dimensional effective action which are order of $O\left(\varphi^{3}\right)$ can be neglected, where $\varphi$ denotes KaluzaKlein modes.

The assumption (i) makes it possible to calculate corrections to the radion potential due to the Casimir effect. In principle, all fields included in the $D$-dimensional theory should contribute to the Casimir effect. However, if $N_{s}$ or $N_{d}$ is large enough then contributions from other fields are small compared with those from the scalar fields and the Dirac fields. Hence, in this limit, the correction to the radion potential is parameterized by only $N_{s}$ and $N_{d}$. In fact, we can show that, after redefining the $D$-dimensional cosmological constant, the corrected potential is parameterized by only two constants: the $D$-dimensional cosmological constant and a particular combination of $N_{s}$ and $N_{d}$.

Therefore, the assumption (i) combined with the assumption (ii) determines the corrected radion potential uniquely up to only one constant. Hence, as we shall show in subsection II d explicitly, the potential is parameterized only by the present value $b_{0}$ of the compactification radius. Since $b_{0}$ can be eliminated from all equations of Kaluza-Klein modes by redefinition of variables, all information about the corrected radion potential can be obtained.

On the other hand, the assumption (iii) makes it possible to investigate each Kaluza-Klein mode independently, provided that kinetic terms and mass terms are properly diagonalized. In this paper, we consider only a particular class of Kaluza-Klein modes corresponding to $D$-dimensional gravitational perturbations. (In Ref. [10] a class of Kaluza-Klein modes corresponding to a $D$-dimensional scalar field was considered.) It is the assumption (iii) that makes it possible to consider these modes independently.

\section{B. Perturbed Einstein-Hilbert action}

We consider the $D$-dimensional Einstein-Hilbert action with a cosmological constant:

$$
I_{E H}=\frac{1}{2 \bar{\kappa}^{2}} \int d^{D} x \sqrt{-\bar{g}}(\bar{R}-2 \bar{\Lambda}),
$$

where $\bar{\kappa}$ is a positive constant, $\bar{R}$ is the $D$-dimensional Ricci scalar, and $\bar{\Lambda}$ is the cosmological constant. We consider a gravitational perturbation $h_{M N}$ around a background metric $\bar{g}_{M N}^{(0)}$, which we shall specify below.

$$
\bar{g}_{M N}=\bar{g}_{M N}^{(0)}+h_{M N} .
$$

By substituting Eq.(2) into Eq.(11), we obtain the perturbed Einstein-Hilbert action as follows.

$$
\begin{aligned}
I_{E H}= & \frac{1}{2 \bar{\kappa}^{2}} \int d^{D} x \sqrt{-\bar{g}^{(0)}}\left[\bar{R}^{(0)}-2 \bar{\Lambda}-h^{M N}\left(\bar{R}_{M N}^{(0)}-\frac{1}{2} \bar{R}^{(0)} \bar{g}_{M N}^{(0)}+\bar{\Lambda} \bar{g}_{M N}^{(0)}\right)\right. \\
& +\frac{1}{8}\left(h^{2}-2 h^{M N} h_{M N}\right) \bar{R}^{(0)}+\frac{1}{2}\left(2 h^{M M^{\prime}} h_{M^{\prime}}^{N}-h h^{M N}\right) \bar{R}_{M N}^{(0)} \\
& +\frac{1}{4}\left\{h_{; M^{\prime}}^{M N}\left(2 h_{M ; N}^{M^{\prime}}-h_{M N}^{; M^{\prime}}\right)+h_{; M}\left(h^{; M}-2 h_{; N}^{M N}\right)\right\} \\
& \left.-\bar{\Lambda}\left(\frac{1}{4} h^{2}-\frac{1}{2} h^{M N} h_{M N}\right)+O\left(h^{3}\right)\right] .
\end{aligned}
$$

where ";" denotes the covariant derivative compatible with $g_{M N}^{(0)}$, and $\bar{R}_{M N}^{(0)}$ and $\bar{R}^{(0)}$ are the Ricci tensor and scalar constructed from $\bar{g}_{M N}^{(0)}$. (See Appendix $\AA$ for a detailed derivation.)

As for the background geometry, we compactify it on a $d$-dimensional sphere $S^{d}(d=D-4)$ :

$$
\bar{g}_{M N}^{(0)} d x^{M} d x^{N}=\hat{g}_{\mu \nu} d x^{\mu} d x^{\nu}+b^{2} \Omega_{i j}^{(d)} d x^{i} d x^{j},
$$

where $\hat{g}_{\mu \nu}$ and $b$ are a 4-dimensional metric and a 4-dimensional scalar that depend only on the four-dimensional coordinate $x^{\mu}(\mu=0,1,2,3)$, and $\Omega_{i j}$ is a metric of the unit $d$-sphere which depends only on the coordinates $x^{i}$ $(i=4, \cdots, D-1)$. Hereafter, we denote $D$-dimensional, 4-dimensional and $d$-dimensional indexes by capital Latin $(M, N, \cdots)$, Greek $(\mu, \nu, \cdots)$ and small Latin $(i, j, \cdots)$ letters, respectively. Note that the scalar $b$ can be interpreted as radius of $S^{d}$.

Since it is convenient to analyze in the so called Einstein frame, we perform the following conformal transformation to make the new frame to be the Einstein frame. 


$$
\hat{g}_{\mu \nu}=\left(\frac{b}{b_{0}}\right)^{-d} g_{\mu \nu}
$$

Note that, if we take $b_{0}$ to be the present value of $b$, then the conformal factor becomes unity after $b$ settles to $b_{0}$. Hence, the change of the frame does not affect the final result, provided that all physical results are interpreted after $b$ settles to $b_{0}$. On the other hand, our analysis becomes much easier if we adopt this conformal transformation. After the conformal transformation, the $D$-dimensional line element is written as

$$
\bar{g}_{M N}^{(0)} d x^{M} d x^{N}=\left(\frac{b}{b_{0}}\right)^{-d} g_{\mu \nu} d x^{\mu} d x^{\nu}+b^{2} \Omega_{i j}^{(d)} d x^{i} d x^{j} .
$$

For this particular background, the gravitational perturbation $h_{M N}$ can be expanded by harmonics on $S^{d}$ as follows

$$
\begin{aligned}
h_{M N} d x^{M} d x^{N}= & \sum_{l m}\left[h_{\mu \nu}^{l m} Y_{l m} d x^{\mu} d x^{\nu}+2\left\{h_{(T) \mu}^{l m}\left(V_{(T) l m}\right)_{i}+h_{(L) \mu}^{l m}\left(V_{(L) l m}\right)_{i}\right\} d x^{\mu} d x^{i}\right. \\
& \left.+\left\{h_{(T)}^{l m}\left(T_{(T) l m}\right)_{i j}+h_{(L T)}^{l m}\left(T_{(L T) l m}\right)_{i j}+h_{(L L)}^{l m}\left(T_{(L L) l m}\right)_{i j}+h_{(Y)}^{l m}\left(T_{(Y) l m}\right)_{i j}\right\} d x^{i} d x^{j}\right],
\end{aligned}
$$

where $Y_{l m}$ is the scalar harmonics function; $V_{(T) l m}$ and $V_{(L) l m}$ are the vector harmonics; $T_{(T) l m}, T_{(L T) l m}$, and $T_{(L L) l m}$ are the tensor harmonics. Here, the coefficients $h_{\mu \nu}^{l m}, h_{(T) \mu}^{l m}, h_{(L) \mu}^{l m}, h_{(T)}^{l m}, h_{(L T)}^{l m}, h_{(L L)}^{l m}$ and $h_{(Y)}^{l m}$ depend only on the four-dimensional coordinates $x^{\mu}$, while the harmonics depend only on the coordinates $x^{i}$ on $S^{d}$. (See Appendix B for definitions and properties of these harmonics.)

Although this expression of $h_{M N}$ includes many terms, some of them represent degrees of freedom of coordinate transformations. In fact, it is shown in Appendix $\square$ that, after gauge-fixing and redefining $g_{\mu \nu}$ and $b$, the perturbation $h_{M N}$ can be expressed as follows.

$$
h_{M N} d x^{M} d x^{N}=\sum_{l m}\left[h_{\mu \nu}^{l m} Y_{l m} d x^{\mu} d x^{\nu}+2 h_{(T) \mu}^{l m}\left(V_{(T) l m}\right)_{i} d x^{\mu} d x^{i}+\left\{h_{(T)}^{l m}\left(T_{(T) l m}\right)_{i j}+h_{(Y)}^{l m}\left(T_{(Y) l m}\right)_{i j}\right\} d x^{i} d x^{j}\right],
$$

where the summations are taken over $l \geq 1$ for the scalar and vector harmonics, and over $l \geq 2$ for the tensor harmonics.

Finally, by substituting this expression into Eq. (3), we obtain the following action.

$$
I_{E H}=I^{(0)}+I^{(1)}+I^{(2)}+O\left(h^{3}\right),
$$

where

$$
\begin{aligned}
I^{(0)} & =\int d^{4} x \sqrt{-g^{(0)}}\left[\frac{1}{2 \kappa^{2}} R^{(0)}-\frac{1}{2} g^{\mu \nu} \partial_{\mu} \sigma \partial_{\nu} \sigma-U_{0}(\sigma)\right], \\
I^{(2)} & =\sum_{l, m} \int d^{4} x \sqrt{-g^{(0)}}\left(\mathcal{L}_{l m}^{(T)}+\mathcal{L}_{l m}^{(V)}+\mathcal{L}_{l m}^{(Y)}\right),
\end{aligned}
$$

and $I^{(1)}$ is linear in $h$. When a total action of the system is considered (see the setting (a) in subsection II A), $I^{(1)}$ should be canceled by other linear terms in the total action because of the equations of motion. Hence, there is no need to consider $I^{(1)}$. The constant $\kappa$, the scalar field $\sigma$, the potential $U_{0}$, and Lagrangian densities $\mathcal{L}_{l m}^{(T, V, Y)}$ are defined as follows. First, the constant $\kappa(>0)$ is defined by

$$
\kappa^{2}=\frac{\bar{\kappa}^{2}}{2^{d} b^{d} \pi}
$$

Next, $\sigma$ is a scalar field defined by

$$
\begin{aligned}
& \sigma=\sigma_{0} \ln \left(\frac{b}{b_{0}}\right), \\
& \sigma_{0}=\sqrt{\frac{d(d+2)}{2 \kappa^{2}}}
\end{aligned}
$$


and is called a radion field [6]. The potential $U_{0}(\sigma)$ of $\sigma$ is given by

$$
U_{0}(\sigma)=\frac{\bar{\Lambda}}{\kappa^{2}} e^{-d \frac{\sigma}{\sigma_{0}}}-\frac{d(d-1)}{2 \kappa^{2} b_{0}^{2}} e^{-(d+2) \frac{\sigma}{\sigma}} .
$$

Finally, the Lagrangian densities $\mathcal{L}_{l m}^{(T, V, Y)}$ are given by

$$
\begin{aligned}
\mathcal{L}_{l m}^{(T)} & =-\frac{1}{2} e^{-4 \frac{\sigma}{\sigma_{0}}} g^{\mu \nu} \partial_{\mu} \chi^{l m} \partial_{\nu} \chi^{l m}-\frac{1}{2} \Lambda_{l m}^{(T)} \chi^{l m} \chi^{l m}, \\
\mathcal{L}_{l m}^{(V)} & =\mathcal{L}_{l m}^{(V)}\left[h_{(T) \mu}^{l m}\right], \\
\mathcal{L}_{l m}^{(Y)} & =\mathcal{L}_{l m}^{(Y)}\left[h_{\mu \nu}^{l m}, h_{(Y)}^{l m}\right],
\end{aligned}
$$

where

$$
\begin{aligned}
\Lambda_{l m}^{(T)}= & e^{-(d+6) \frac{\sigma}{\sigma_{0}}}\{l(l+d-1)+(d-1)(d-2)\} b_{0}^{-2} \\
& +e^{-4 \frac{\sigma}{\sigma_{0}}}\left[(d+4) \nabla^{2}\left(\frac{\sigma}{\sigma_{0}}\right)-\left\{\frac{d(d+2)}{2}-4\right\} \nabla\left(\frac{\sigma}{\sigma_{0}}\right)^{2}+\left\{R^{(0)}-2 e^{-d \frac{\sigma}{\sigma_{0}}} \bar{\Lambda}\right\}\right], \\
\chi^{l m} \equiv & \sqrt{\frac{b_{0}^{-4}}{2^{d+2} \pi \kappa^{2}}} h_{(T)}^{l m} \quad(l \geq 2) .
\end{aligned}
$$

(Since hereafter we analyze $h_{(T)}^{l m}\left(\right.$ or $\left.\chi^{l m}\right)$ only, we have not written down explicit form of $\mathcal{L}_{l m}^{(V)}$ and $\mathcal{L}_{l m}^{(Y)}$.) Hence, up to the second order in Kaluza-Klein modes, $h_{(T)}^{l m}\left(\right.$ or $\left.\chi^{l m}\right)$ is decoupled from all other Kaluza-Klein modes. (See the assumption (iii) in subsection II A.) In this paper, for simplicity, we investigate the parametric resonance of $\chi^{l m}$

$\left(\right.$ or $h_{(T)}^{l m}$ ) only. It should be stressed again that it is the assumption (iii) in subsection II A that makes it possible to consider these modes independently.

Here, note that the constant $b_{0}$ is arbitrary at the moment and that we can eliminate it from all equations. However, in the next subsection, we chose $b_{0}$ to be the present value of $b$.

\section{Stabilization of the radion potential}

Here we assume that the Casimir effect [7] stabilizes the radion potential. In other words, we assume that quantum corrections to $U_{0}$ stabilize compactification. Thanks to the assumptions (i) and (ii) in subsection II A, we can easily calculate the stabilized radion potential, which we shall denote by $U_{1}$.

First, for all Kaluza-Klein modes of $N_{s}$ scalar fields and $N_{d}$ Dirac fields, mass in the Einstein frame is proportional to the common quantity.

$$
M^{2} \propto b_{0}^{-2} e^{-(d+2) \sigma / \sigma_{0}} .
$$

Hence, because of the assumption (i), we can obtain the following 1-loop effective potential. (See, for example, Ref. 10] for details.)

$$
V_{1 \text { loop }}(\sigma)=C e^{-2(d+2) \sigma / \sigma_{0}},
$$

where $C$ is a constant determined by $N_{s}$ and $N_{d}$. There are corrections to the $D$-dimensional cosmological constant $\bar{\Lambda}$, too. However, these corrections can be absorbed in $\bar{\Lambda}$ by redefining $\bar{\Lambda}$. Hence, the corrected radion potential $U_{1}$ is given by

$$
U_{1}(\sigma)=U_{0}(\sigma)+V_{1 \text { loop }}(\sigma)
$$

Next, the assumption (ii) requires that the minimum of $U_{1}$ should be zero. Hence, $\bar{\Lambda}$ is determined as follows.

$$
\bar{\Lambda}=\frac{d(d-1)(d+2)}{2(d+4) b_{0}^{2}},
$$

where we have specified the constant $b_{0}$ as 


$$
b_{0}=\sqrt{\frac{d(d-1)}{(d+4) C \kappa^{2}}} .
$$

Thus, we obtain the following radion potential [11, 10].

$$
U_{1}(\sigma)=\alpha\left[\frac{2}{d+2} e^{-2(d+2) \sigma / \sigma_{0}}+e^{-d \sigma / \sigma_{0}}-\frac{d+4}{d+2} e^{-(d+2) \sigma / \sigma_{0}}\right],
$$

where $\alpha$ is a constant given by

$$
\alpha=\frac{d(d-1)(d+2)}{2(d+4) \kappa^{2} b_{0}^{2}} .
$$

This potential has the minimum zero at $\sigma=0$, provided that $d \geq 2$. Thus, in this case, the 4-dimensional cosmological constant is actually zero and $b_{0}$ is the present value of $b$. If $d=1$ then the potential is completely flat. Hence, hereafter, we assume that $d \geq 2$.

It is notable that the radion potential has been determined uniquely up to the present value $b_{0}$ of the compactification radius $b$. Since $b_{0}$ can be eliminated from all equations of $\chi^{l m}$ by redefinition of variables, all information about the corrected radion potential has been obtained.

Finally, the perturbed Einstein-Hilbert action corrected by the Casimir effect is given by Eq. (9), provided that $U_{0}$ is replaced by $U_{1}$ given by Eq. (26). Therefore, hereafter, we investigate the dynamics of the field $\chi^{l m}$ coupled to the radion field $\sigma$ in the expanding universe. These fields are described by the following action.

$$
I=I_{\sigma}+I_{\chi}
$$

where

$$
\begin{aligned}
& I_{\sigma}=-\int d^{4} x \sqrt{-\bar{g}^{(0)}}\left[\frac{1}{2} g^{\mu \nu} \partial_{\mu} \sigma \partial_{\nu} \sigma+U_{1}(\sigma)\right], \\
& I_{\chi}=-\frac{1}{2} \int d^{4} x \sqrt{-\bar{g}^{(0)}}\left[e^{-4 \frac{\sigma}{\sigma_{0}}} g^{\mu \nu} \partial_{\mu} \chi^{l m} \partial_{\nu} \chi^{l m}+\Lambda_{l m}^{(T)} \chi^{l m} \chi^{l m}\right] .
\end{aligned}
$$

Here, we mention that in the total action there may be other terms which are second order in $\chi^{l m}$. In fact, there should appear other mass terms for $\chi^{l m}$ since some zero modes may couple to $\chi^{l m}$. However, providing the assumption (vii) in the next section, these additional mass terms are small enough since zero modes are considered as usual lowenergy matter whose energy density and pressure are bounded from above by 4-dimensional cosmological parameters. Therefore, the above action is enough for our purpose.

\section{PARAMETRIC RESONANCE}

In this section we investigate parametric resonance of the field $\chi^{l m}$ due to the oscillation of the radion field $\sigma$ in the expanding universe. For this purpose, we specify 4-dimensional geometry and initial condition somewhat.

(iv) The background 4-dimensional geometry is expressed by the flat FRW metric, and the radion field is homogeneous in the FRW universe.

(v) Initially, there is no excitation of Kaluza-Klein modes. Thus, the FRW universe is driven by the radion field as well as by usual homogeneous matter corresponding to zero modes.

Since the radion potential $U_{1}(\sigma)$ has exponential terms, it seems difficult to analyze oscillation of $\sigma$ with large amplitude analytically. Hence, we assume that the amplitude of the oscillation is small enough. Moreover, for further simplicity, we assume that the time scale of the cosmological expansion is much longer than the time scale of the radion oscillation. Namely, we assume the following.

(vi) We assume that the amplitude of the radion oscillation around $\sigma=0$ is small compared with $\sigma_{0}$.

(vii) The energy scale of the cosmological expansion is much lower than that of compactification: we assume that $H b_{0} \ll 1$ and $\dot{H} b_{0}^{2} \ll 1$, where $H \equiv \dot{a} / a$ and the dot denotes the derivative with respect to the cosmological time.

Note that the assumption (vi) leads to the so called narrow resonance regime for the parametric resonance. 


\section{A. FRW background and homogeneous radion}

According to the assumptions (iv) and (v), we consider the homogeneous radion field and the flat FRW universe, which is driven by the radion field and usual matter corresponding to zero modes:

$$
\begin{aligned}
g_{\mu \nu} d x^{\mu} d x^{\nu} & =-d t^{2}+a(t)^{2}\left(d x^{2}+d y^{2}+d z^{2}\right), \\
\sigma & =\sigma(t) .
\end{aligned}
$$

The cosmological evolution equations are

$$
\begin{aligned}
H^{2} & =\frac{8 \pi G_{N}}{3}\left(\rho_{\sigma}+\rho_{0}\right) \\
\dot{H}+H^{2} & =-\frac{4 \pi G_{N}}{3}\left[\left(\rho_{\sigma}+\rho_{0}\right)+3\left(p_{\sigma}+p_{0}\right)\right],
\end{aligned}
$$

where the subscripts ' $\sigma$ ' and ' 0 ' mean contributions from the radion $\sigma$ and those from usual matter corresponding to zero modes, respectively. Here, $H \equiv \dot{a} / a, G_{N}=\kappa^{2} / 8 \pi$, and the dot denotes derivative with respect to the time $t$.

Now, because of the symmetry of the background, it is convenient to analyze the field $\chi^{l m}$ in the momentum space. Hence, we decompose the field $\chi^{l m}$ as follows:

$$
\chi_{l m}=a^{-\frac{3}{2}} \int \frac{\sqrt{2} d^{3} \boldsymbol{k}}{(2 \pi)^{3}}\left[\chi_{l m}^{(1)}(t) \cos (\boldsymbol{k} \cdot \boldsymbol{x})+\chi_{l m \boldsymbol{k}}^{(2)}(t) \sin (\boldsymbol{k} \cdot \boldsymbol{x})\right],
$$

where $\chi_{\operatorname{lm} \boldsymbol{k}}^{(1,2)}$ are real functions of the cosmological time $t$ and the vector $\boldsymbol{x}$ represents $(x, y, z)$. Correspondingly, the action $I_{\chi}$ is rewritten as

$$
\begin{aligned}
& I_{\chi}=\int d t L_{\chi} \\
& L_{\chi}=\frac{1}{2} \sum_{l, m} \sum_{i=1,2} \int d^{3} \boldsymbol{k} e^{-4 \frac{\sigma}{\sigma_{0}}}\left[\left\{\partial_{t} \chi_{l m \boldsymbol{k}}^{(i)}(t)\right\}^{2}-m^{2}(t)\left\{\chi_{l m \boldsymbol{k}}^{(i)}(t)\right\}^{2}\right]
\end{aligned}
$$

where

$$
m^{2}(t)=\Lambda_{l m}^{(T)}+e^{-4 \frac{\sigma}{\sigma_{0}}} \frac{\boldsymbol{k} \cdot \boldsymbol{k}}{a^{2}}-\left\{\frac{4}{9} H^{2}+\frac{3}{2} \dot{H}-6 H\left(\frac{\dot{\sigma}}{\sigma_{0}}\right)\right\} .
$$

Although the kinetic term in the above Lagrangian has time dependence, we can eliminate it by using a new time variable $\tau$ defined by

$$
\frac{d \tau}{d t}=\exp \left[4 \frac{\sigma(t)}{\sigma_{0}}\right]
$$

In fact, the equations of motion for $\chi_{l m \boldsymbol{k}}^{(1,2)}$ are written as

$$
\frac{\partial^{2} Q}{\partial \tau^{2}}+\Omega^{2}(\tau) Q=0
$$

where $Q$ denotes $\chi_{l m \boldsymbol{k}}^{(1,2)}$ and $\Omega$ is defined by

$$
\Omega^{2}(\tau)=m^{2}(t(\tau)) \exp \left[-4 \frac{\sigma(t(\tau))}{\sigma_{0}}\right] .
$$

The hamiltonian with respect to the time $\tau$ is

$$
\mathcal{H} \equiv \frac{f}{2}\left(P^{2}+\Omega^{2} Q^{2}\right),
$$

where $P$ is the momentum conjugate to $Q$.

After the oscillation of $\sigma$ ends up at $\sigma=0$, the right hand side of Eq. (35) becomes unity. Thus, after the end of the oscillation, the hamiltonian $\mathcal{H}$ with respect to $\tau$ coincides with the hamiltonian with respect to the original cosmological time $t$. At the same time, those hamiltonians become conserved quantities. Hence, we can analyze the excitation of $\chi_{l m \boldsymbol{k}}^{(1,2)}$ by using the new time variable $\tau$. 


\section{B. Small oscillation of the radion}

Providing the assumptions (vi) and (vii), the equation of motion for $\sigma$ gives

$$
\frac{\sigma(t)}{\sigma_{0}}=\tilde{\sigma}(t) \cos \omega\left(t-t_{0}\right),
$$

where $t_{0}$ is a constant, $\omega$ is defined by

$$
\omega=\frac{1}{2} \sqrt{U_{1}^{\prime \prime}(0)}=\frac{1}{b_{0}} \sqrt{\frac{d-1}{2}},
$$

and $\tilde{\sigma}(t)(\ll 1)$ is a slowly varying amplitude:

$$
\tilde{\sigma}(t) \propto a^{-3 / 2}
$$

Now, by using Eq. (39), we can express $\Omega^{2}$ in terms of the new time variable $\tau$ up to the first order in $\tilde{\sigma}$ :

$$
\Omega^{2}(\tau)=\omega^{2}\left[A+B+\tilde{\epsilon} \cos \left\{2 \omega\left(\tau-\tau_{0}\right)\right\}+O\left(\tilde{\epsilon}^{2}\right)\right]
$$

where $\tau_{0}$ is a constant and

$$
\begin{aligned}
A & =\frac{2 b_{0}{ }^{2}\left(\boldsymbol{k}^{2} / a\right)}{d-1}-\omega^{2}\left(\frac{9}{4} H^{2}+\frac{3}{2} \dot{H}\right), \\
B & =\frac{2(d+4)\{l(l+d-1)+(d-2)(d-1)\}-d(d-1)(d+2)}{b_{0}^{2} \omega^{2}} \\
\tilde{\epsilon} & =\tilde{\sigma}\left\{(d-1) \frac{d(d+2)(d+8)-l(l+d-1)(d-2)(d+10)}{b_{0}^{2} \omega^{2}}-\frac{8}{\omega^{2}}\left(\boldsymbol{k}^{2} / a\right)^{2}+4(d+4)\right\} .
\end{aligned}
$$

The first term in $A$ is essentially the squared momentum along the three-dimensional spatial directions divided by $\omega^{2}, B$ is essentially the squared momentum along $S^{d}$ divided by $\omega^{2}$, and $\tilde{\epsilon}$ is the properly normalized amplitude of the radion oscillation.

\section{Result}

The expression (42) is of almost the same form as the expression of $\Omega^{2}$ investigated in Ref. [10]. Hence, we can analyze the parametric resonance in the same way as in Ref. 110. Thus, the necessary and sufficient condition for the catastrophic creation of quanta of the Kaluza-Klein mode is

$$
\begin{aligned}
B & \leq 1 \\
|B-1| & =O(\tilde{\epsilon}) .
\end{aligned}
$$

However, it is easily shown that

$$
B>3
$$

for any values of $d(\geq 2)$ and $l(\geq 2)$. (See the setting (c) in subsection II A.) Therefore, it is concluded that quanta of the field $\chi^{l m}$ are not overproduced by the parametric resonance due to the small oscillation of the radion $\sigma$.

\section{SUMMARY AND DISCUSSIONS}

We have investigated excitation of Kaluza-Klein modes due to the parametric resonance caused by oscillation of radius of compactification in the narrow resonance regime. In particular, we have considered a gravitational perturbation around a $D$-dimensional spacetime compactified on a $(D-4)$-sphere. Among many Kaluza-Klein modes of the gravitational perturbation we have investigated part of Kaluza-Klein modes which correspond to massive scalar 
fields in 4-dimension. We have obtained that quanta of these modes are not excited so catastrophically in the narrow resonance regime.

Now, several comments are in order.

First, we discuss about the consistency among the assumption (v), (vi) and (vii). Energy density $\rho_{\sigma}$ and pressure $p_{\sigma}$ of the radion is of the following order.

$$
\rho_{\sigma} \sim p_{\sigma} \sim \tilde{\epsilon}^{2} b_{0}^{-2} \kappa^{-2}
$$

where $\tilde{\epsilon} \sim \sigma / \sigma_{0}$. Thus, the condition $H^{2} \sim \kappa^{2} \rho_{\sigma}$ can be satisfied, even if $\tilde{\epsilon} \ll 1, H b_{0} \ll 1$ and $\dot{H} b_{0}^{2} \ll 1$. In other words, the three assumptions (v), (vi) and (vii) can be satisfied simultaneously.

Next, we discuss about validity of our strategy. Although we have investigated only a particular class of KaluzaKlein modes in this paper, in principle we have to investigate all Kaluza-Klein modes in order to judge whether a way of compactification is acceptable or not. If we find that one of these many Kaluza-Klein modes is excited abundantly, then the way of compactification should be rejected. In this respect, there may be an embarrassing expectation: it is easily expected that, even if we find that $N_{K K} \gg 1$, the result will be significantly changed by backreaction, where $N_{K K}$ is the number of created quanta of a Kaluza-Klein mode. In fact, by the energy conservation and the assumptions (v) and (vii), it is easily concluded that $N_{K K} \ll 1$. Thus, it might be expected that under the assumptions (v) and (vii) we might not be able to judge whether a way of compactification is acceptable or not. However, this embarrassing consideration does not spoil our analysis. Actually, if we obtain $N_{K K} \gg 1$ by ignoring the backreaction, then this overestimating result implies at least that $\rho_{K K}$ is not negligible compared with $\rho_{\sigma}$ since the backreaction becomes effective only when $\rho_{K K}$ is not negligible compared with $\rho_{\sigma}$, where $\rho_{K K}$ is energy density of the Kaluza-Klein mode. Therefore, providing the assumption (v), the result $N_{K K} \gg 1$ obtained by ignoring the backreaction implies that $\rho_{K K}$ is not negligible compared with energy density of radiation, which should be rejected as explained in Sec. I. Because of almost the same reason, we can justify the assumption (iii). First, provided that the higher-order coupling constant is at most of order unity in the unit of $b_{0}$, those higher-order terms should be inefficient as long as $\rho_{K K} \ll b_{0}^{-4}$. Physically, this statement is almost equivalent to the statement that sufficiently small oscillation does not feel any higher-order corrections to its potential. Therefore, we can neglect higher-order terms if the created number of Kaluza-Klein quanta is small. (See the assumption (v) for the initial condition.) In other words, if higher-order terms becomes efficient at some epoch, then it implies that the number of Kaluza-Klein quanta created so far is not small, and such a situation should be rejected as explained in Sec. I.

Finally, we comment on possible future works. (A) We have to investigate Kaluza-Klein modes of other fields as well as other gravitational modes. (B) We have to take inflation into consideration. (C) If we can extend our analysis to the broad resonance regime, then it will give stronger constraints on ways of compactification. Note that, even in the broad resonance regime, Eqs. (36) and (38) can be used. (D) Other mechanisms of compactification might deserve considering, for example, the monopole-like configuration of an anti-symmetric field [12]. (E) It might be also interesting to investigate the parametric resonance of Kaluza-Klein modes in the Randall-Sundrum scenario [- 4 ] with stabilized radion [13].

\section{ACKNOWLEDGMENTS}

KU would like to thank Professor H. Kodama, Professor M. Sasaki, Professor M. Sakagami, Professor J. Soda, S. Tsujikawa, K. Koyama for discussions. KU would also like to thank K. Yamashita for continuing encouragement. YM would like to thank Professor T. Nakamura for continuous encouragement. SM would like to thank Professor W. Israel for continuing encouragement and Professor L. Kofman for his warm hospitality in Canadian Institute for Theoretical Astrophysics. SM's work is supported by the CITA National Fellowship and the NSERC operating research grant.

\section{APPENDIX A: SECOND-ORDER EINSTEIN ACTION}

The purpose of this appendix is to expand the Einstein-Hilbert action by $h_{M N}$ up to the second order. Although in the main part of this paper all quantities defined in $D$-dimension have " $"$ ", we omit it in this for simplicity.

Let us decompose the metric $g_{M N}$ into the background $g_{M N}^{(0)}$ and perturbation $h_{M N}$ :

$$
g_{M N}=g_{M N}^{(0)}+h_{M N} .
$$

Hereafter, we use the background metric $g_{M N}^{(0)}$ to raise the indices of $h_{M N}$ : 


$$
\begin{aligned}
h^{M}{ }_{N} & =h_{N}{ }^{M} \equiv g^{M O} h_{O N}, \\
h^{M N} & \equiv g^{(0) M O} g^{(0) N P} h_{O P}, \\
h & \equiv g^{(0) M N} h_{M N} .
\end{aligned}
$$

First, we can easily expand $g^{M N}, \sqrt{-g}$ and the Christoffel symbol $\Gamma_{M N}^{P}$ as follows, where $g$ is the determinant of $g_{M N}$.

$$
\begin{aligned}
& g^{M N}=g^{M N(0)}-h^{M N}+h^{M O} h_{O}{ }^{N}+O\left(h^{3}\right), \\
& \sqrt{-g}=\sqrt{-g^{(0)}}\left\{1+\frac{1}{2} h+\frac{1}{2}\left(\frac{1}{4} h^{2}-\frac{1}{2} h^{M N} h_{M N}\right)\right\}+O\left(h^{3}\right), \\
& \Gamma_{M N}^{P}=\Gamma_{M N}^{(0)}{ }_{M N}^{P}+\Gamma^{(1)}{ }_{M N}^{P}+\Gamma_{M N}^{(2)}{ }_{M}^{P}+O\left(h^{3}\right),
\end{aligned}
$$

where $g^{(0)}$ and $\Gamma^{(0)}{ }_{M N}^{P}$ are the determinant and the Christoffel symbol of the background metric $g_{M N}^{(0)}$, and

$$
\begin{aligned}
& \Gamma_{M N}^{(1)}{ }_{M N} \equiv \frac{1}{2} g^{(0)}{ }^{P Q}\left(h_{Q M, N}+h_{Q N, M}-h_{M N}, Q\right), \\
& \Gamma_{M N}^{(2)}{ }_{M N} \equiv-\frac{1}{2} h^{P Q}\left(h_{Q M, N}+h_{Q N, M}-h_{M N}, Q\right) .
\end{aligned}
$$

Here, ", " denotes the partial derivative.

Next, the Ricci tensor is expanded as follows.

$$
\begin{aligned}
R_{M N} & =R_{M P N}^{P}=\Gamma_{M N, P}^{P}-\Gamma_{M P, N}^{P}+\Gamma_{S P}^{P} \Gamma_{M N}^{S}-\Gamma_{S N}^{P} \Gamma_{M P}^{S} \\
& =R_{M N}^{(0)}+R_{M N}^{(1)}+R_{M N}^{(2)}+O\left(h^{3}\right),
\end{aligned}
$$

where $R_{M N}^{(0)}$ is the Ricci tensor for the background metric $g_{M N}^{(0)}$, and

$$
\begin{aligned}
& R_{M N}^{(1)}=\Gamma_{M N, P}^{(1)}-\Gamma_{M P, N}^{(1)}{ }_{M P}^{P}, \\
& R_{M N}^{(2)}=\Gamma_{M N, P}^{(2)}-\Gamma_{M P, N}^{(2)}{ }_{M P}^{P}+\Gamma^{(1)}{ }_{S P}^{P} \Gamma^{(1)}{ }_{M N}^{S}-\Gamma^{(1)}{ }_{S N}^{P} \Gamma^{(1)}{ }_{M P}^{S} .
\end{aligned}
$$

Therefore, we obtain the following expansion.

$$
\sqrt{-g} R=\sqrt{-g} g^{M N} R_{M N}=\sqrt{-g^{0}}\left(R^{(0)}+R^{(1)}+R^{(2)}+O\left(h^{3}\right)\right)
$$

where $R^{(0)}$ is the Ricci scalar for the background metric $g_{M N}^{(0)}$, and

$$
\begin{aligned}
R^{(1)} \equiv & \frac{1}{2} h R^{(0)}-h^{M N} R_{M N}^{(0)}+g^{(0)^{M N}} R^{(1)}{ }_{M N} \\
= & -h^{M N}\left(R_{M N}^{(0)}-\frac{1}{2} R^{(0)} g_{M N}\right)+\left(h^{M N}{ }_{; N}-h^{; M}\right)_{; M}, \\
R^{(2)} \equiv & \frac{1}{2}\left(\frac{1}{4} h^{2}-\frac{1}{2} h^{P Q} h_{P Q}\right) R^{(0)}+h^{M P} h_{P}{ }^{N} R_{M N}^{(0)}+g^{(0)}{ }^{M N} R^{(2)}{ }_{M N} \\
& -\frac{1}{2} h h^{M N} R_{M N}^{(0)}-h^{M N} R_{M N}^{(1)}+\frac{1}{2} h g^{(0)^{M N}} R_{M N}^{(1)} \\
= & \frac{1}{8}\left(h^{2}-2 h^{P Q} h_{P Q}\right) R^{(0)}+\frac{1}{2}\left(2 h^{M P} h_{P}{ }^{N}-h h^{M N}\right) R_{M N}^{(0)} \\
& +\frac{1}{4}\left\{h_{; P}^{M N}+\left(2 h^{P}{ }_{M} ; h_{M N} ; P\right)+h_{; M}\left(h^{; M}-2 h^{M N} ; N\right)\right\}+X_{; P}^{P} .
\end{aligned}
$$

Here, $X^{P}$ is a vector constructed from $h_{M N}$, and ";" denotes the covariant derivative compatible with the background metric $g_{M N}^{(0)}$.

Finally, by using the above expressions and integrating by part, we obtain the perturbed Einstein-Hilbert action up to the second order in $h_{M N}$. 


$$
\begin{aligned}
I_{E H} \equiv & \frac{1}{2 \kappa^{2}} \int d^{D} x \sqrt{-g}\left(R^{(0)}-2 \Lambda\right) \\
= & \frac{1}{2 \kappa^{2}} \int d^{D} x \sqrt{-g^{(0)}}\left[R^{(0)}-2 \Lambda-h^{M N}\left(R_{M N}^{(0)}-\frac{1}{2} R^{(0)} g_{M N}^{(0)}+\Lambda g_{M N}^{(0)}\right)\right. \\
& +\frac{1}{8}\left(h^{2}-2 h^{M N} h_{M N}\right) R^{(0)}+\frac{1}{2}\left(2 h^{M M^{\prime}} h_{M^{\prime}}^{N}-h h^{M N}\right) R_{M N}^{(0)} \\
& +\frac{1}{4}\left\{h_{; M^{\prime}}^{M N}\left(2 h_{M ; N}^{M^{\prime}}-h_{M N} ; M^{\prime}\right)+h_{; M}\left(h^{; M}-2 h_{; N}^{M N}\right)\right\} \\
& \left.-\Lambda\left(\frac{1}{4} h^{2}-\frac{1}{2} h^{M N} h_{M N}\right)+O\left(h^{3}\right)\right] .
\end{aligned}
$$

\section{APPENDIX B: HARMONICS ON D-SPHERE}

In this Appendix we give definitions and basic properties of scalar, vector and tensor harmonics on a unit $d$ sphere [14. Throughout this Appendix we will use the notation that $\Omega_{i j}$ is the metric of the unit $d$-sphere and $D$ is the covariant derivative compatible with $\Omega_{i j}$.

\section{1. scalar harmonics}

The scalar harmonics is supposed to satisfy the following relations.

$$
\begin{gathered}
D^{2} Y_{l m}+l(l+d-1) Y_{l m}=0 \quad(l \geq 0), \\
\int d^{d} x \sqrt{\Omega} Y_{l m} Y_{l^{\prime} m^{\prime}}=\delta_{l l^{\prime}} \delta_{m m^{\prime}} .
\end{gathered}
$$

\section{2. vector harmonics}

First, in general, a vector field $V$ on the unit $d$-sphere can be decomposed as

$$
V_{i}=V_{(T) i}+\partial_{i} f
$$

where $f$ is a function and $V_{(T)}$ is a transverse vector field:

$$
D^{i}\left(V_{(T)}\right)_{i}=0
$$

Thus, the vector field $V_{i}$ can be expanded by using the scalar harmonics $Y_{l m}$ and transverse vector harmonics $V_{(T) l m}$ as

$$
V_{i}=\sum_{l m}\left[c_{(T)}^{l m}\left(V_{(T) l m}\right)_{i}+c_{(L)}^{l m} \partial_{i} Y_{l m}\right]
$$

where $c_{(T)}^{l m}$ and $c_{(L)}^{l m}$ are constants, and the transverse vector harmonics $V_{(T) l m}(l \geq 1)$ is supposed to satisfy the following relations.

$$
\begin{aligned}
D^{2} V_{(T) l m}+\{l(l+d-1)-1\} V_{(T) l m} & =0 \\
D^{i}\left(V_{(T) l m}\right)_{i} & =0 \\
\int d^{d} x \sqrt{\Omega} \Omega^{i j}\left(V_{(T) l m}\right)_{i}\left(V_{(T) l^{\prime} m^{\prime}}\right)_{j} & =\delta_{l l^{\prime}} \delta_{m m^{\prime}} .
\end{aligned}
$$

¿From Eq. (B5), it is convenient to define longitudinal vector harmonics $V_{(L) l m}$ by

$$
\left(V_{(L) l m}\right)_{i}=\partial_{i} Y_{l m} \quad(l \geq 1) .
$$


It is easily shown that the longitudinal vector harmonics satisfies the following properties.

$$
\begin{aligned}
D^{2} V_{(L) l m}+\{l(l+d-1)-(d-1)\} V_{(L) l m} & =0, \\
D^{i}\left(V_{(L) l m}\right)_{i} & =-l(l+d-1) Y_{l m}, \\
D_{[i}\left(V_{(L) l m}\right)_{j]} & =0, \\
\int d^{d} x \sqrt{\Omega} \Omega^{i j}\left(V_{(L) l m}\right)_{i}\left(V_{(L) l^{\prime} m^{\prime}}\right)_{j} & =l(l+d-1) \delta_{l l^{\prime}} \delta_{m m^{\prime}}, \\
\int d^{d} x \sqrt{\Omega} \Omega^{i j}\left(V_{(T) l m}\right)_{i}\left(V_{(L) l^{\prime} m^{\prime}}\right)_{j} & =0 .
\end{aligned}
$$

\section{Tensor harmonics}

First, in general, a symmetric second-rank tensor field $T_{i j}$ can be decomposed as

$$
T_{i j}=T_{(T) i j}+D_{i} V_{j}+D_{j} V_{i}+f \Omega_{i j}
$$

where $f$ is a function, $V_{i}$ is a vector field and $T_{(T)} i j$ is a transverse traceless symmetric tensor field:

$$
\begin{aligned}
\left(T_{(T)}\right)_{i}^{i} & =0, \\
D^{i}\left(T_{(T)}\right)_{i j} & =0 .
\end{aligned}
$$

Thus, the tensor field $T_{i j}$ can be expanded by using the vector harmonics $V_{(T) l m}$ and $V_{(L) l m}$, and transverse vector harmonics $V_{(T)} l m$ as

$$
\begin{aligned}
T_{i j}= & \sum_{l m}\left[c_{(T)}^{l m}\left(T_{(T) l m}\right)_{i j}+c_{(L T)}^{l m}\left\{D_{i}\left(V_{(T) l m}\right)_{j}+D_{j}\left(V_{(T) l m}\right)_{i}\right\}\right. \\
& \left.+c_{(L L)}^{l m}\left\{D_{i}\left(V_{(L) l m}\right)_{j}+D_{j}\left(V_{(L) l m}\right)_{i}\right\}+c_{(Y)}^{l m} Y_{l m} \Omega_{i j}\right]
\end{aligned}
$$

where $c_{(T)}^{l m}, c_{(L T)}^{l m}, c_{(L L)}^{l m}$ and $c_{(Y)}^{l m}$ are constants, and the transverse tensor harmonics $T_{(T) l m}(l \geq 2)$ is supposed to satisfy the following relations.

$$
\begin{aligned}
D^{2} T_{(T) l m}+\{l(l+d-1)-2\} T_{(T) l m} & =0 \\
\left(T_{(T) l m}\right)_{i}^{i} & =0, \\
D^{i}\left(T_{(T) l m}\right)_{i j} & =0, \\
\int d^{d} x \sqrt{\Omega} \Omega^{i i^{\prime}} \Omega^{j j^{\prime}}\left(T_{(T) l m}\right)_{i j}\left(T_{(T) l^{\prime} m^{\prime}}\right)_{i^{\prime} j^{\prime}} & =\delta_{l l^{\prime}} \delta_{m m^{\prime}} .
\end{aligned}
$$

From Eq. (B11), it is convenient to define tensor harmonics $T_{(L T) l m}, T_{(L L) l m}$, and $T_{(Y) l m}$ by

$$
\begin{aligned}
\left(T_{(L T) l m}\right)_{i j} & \equiv D_{i}\left(V_{(T) l m}\right)_{j}+D_{j}\left(V_{(T) l m}\right)_{i}, \quad(l \geq 2) \\
\left(T_{(L L) l m}\right)_{i j} & \equiv D_{i}\left(V_{(L) l m}\right)_{j}+D_{j}\left(V_{(L) l m}\right)_{i}-\frac{2}{d} \Omega_{i j} D^{k}\left(V_{(L) l m}\right)_{k}, \quad(l \geq 2) \\
\left(T_{(Y) l m}\right)_{i j} & \equiv \Omega_{i j} Y_{(Y) l m} \quad(l \geq 0) .
\end{aligned}
$$

It is easily shown that these tensor harmonics satisfy the following properties.

$$
\begin{gathered}
D^{2} T_{(L T) l m}+\{l(l+d-1)-(d+2)\} T_{(L T) l m}=0, \\
D^{i}\left(T_{(L T) l m}\right)_{i j}=-\{l(l+d-1)-d\}\left(V_{(T) l m}\right)_{j}, \\
\left(T_{(L T) l m}\right)_{i}^{i}=0, \\
D^{2} T_{(L L) l m}+\{l(l+d-1)-2 d\} T_{(L L) l m}=0, \\
D^{i}\left(T_{(L L) l m}\right)_{i j}=-\frac{2(d-1)}{d}\{l(l+d-1)-d\}\left(V_{(L) l m}\right)_{j}, \\
\left(T_{(L L) l m}\right)_{i}^{i}=0,
\end{gathered}
$$


and

$$
\begin{aligned}
D^{2} T_{(Y) l m}+l(l+d-1) T_{(Y) l m} & =0, \\
D^{i}\left(T_{(Y) l m}\right)_{i j} & =\left(V_{(L) l m}\right)_{j}, \\
\left(T_{(Y) l m}\right)_{i}^{i} & =d Y_{l m} .
\end{aligned}
$$

It is also easy to show the following formulas of integral as well as the orthogonality between different types of tensor harmonics.

$$
\begin{aligned}
& \int d^{d} x \sqrt{\Omega} \Omega^{i i^{\prime}} \Omega^{j j^{\prime}}\left(T_{(L T) l m}\right)_{i j}\left(T_{(L T) l^{\prime} m^{\prime}}\right)_{i^{\prime} j^{\prime}}=2\{l(l+d-1)-d\} \delta_{l l^{\prime}} \delta_{m m^{\prime}}, \\
& \int d^{d} x \sqrt{\Omega} \Omega^{i i^{\prime}} \Omega^{j j^{\prime}}\left(T_{(L L) l m}\right)_{i j}\left(T_{(L L)} l^{\prime} m^{\prime}\right)_{i^{\prime} j^{\prime}}=\frac{4 l(l+d-1)(d-1)}{d}\{l(l+d-1)-d\} \delta_{l l^{\prime}} \delta_{m m^{\prime}}, \\
& \int d^{d} x \sqrt{\Omega} \Omega^{i i^{\prime}} \Omega^{i i^{\prime}}\left(T_{(Y) l m}\right)_{i j}\left(T_{(Y) l^{\prime} m^{\prime}}\right)_{i^{\prime} j^{\prime}}=d \delta_{l l^{\prime}} \delta_{m m^{\prime}}
\end{aligned}
$$

\section{Integration formulas}

Finally, we list up some other formulas of integration.

$$
\begin{aligned}
& \int d^{d} x \sqrt{\Omega} D^{i}\left(V_{(T) l m}\right)_{j} D_{i}\left(V_{(T) l^{\prime} m^{\prime}}\right)^{j}=\{l(l+d-1)-1\} \delta_{l l^{\prime}} \delta_{m m^{\prime}}, \\
& \int d^{d} x \sqrt{\Omega} D^{i}\left(V_{(T) l m}\right)_{j} D_{i}\left(V_{(T) l^{\prime} m^{\prime}}\right)^{j}=-(d-1) \delta_{l l^{\prime}} \delta_{m m^{\prime}}, \\
& \int d^{d} x \sqrt{\Omega} D^{i}\left(T_{(T) l m}\right)^{j k} D_{i}\left(T_{(T) l^{\prime} m^{\prime}}\right)_{j k}=\{l(l+d-1)-2\} \delta_{l l^{\prime}} \delta_{m m^{\prime}}, \\
& \int d^{d} x \sqrt{\Omega} D^{i}\left(T_{(Y) l m}\right)^{j k} D_{i}\left(T_{(Y) l^{\prime} m^{\prime}}\right)_{j k}=d\{l(l+d-1)-2\} \delta_{l l^{\prime}} \delta_{m m^{\prime}}, \\
& \int d^{d} x \sqrt{\Omega} D^{k}\left(T_{(T) l m}\right)^{i j} D_{i}\left(T_{(T) l^{\prime} m^{\prime}}\right)_{j k}=-d \delta_{l l^{\prime}} \delta_{m m^{\prime}}, \\
& \int d^{d} x \sqrt{\Omega} D^{k}\left(T_{(Y) l m}\right)^{i j} D_{i}\left(T_{(Y) l^{\prime} m^{\prime}}\right)_{j k}=l(l+d-1) \delta_{l l^{\prime}} \delta_{m m^{\prime}} .
\end{aligned}
$$

\section{APPENDIX C: GAUGE FIXING}

In this appendix we show that there is a particular choice of gauge in which the gravitational perturbation $h_{M N}$ is expanded as Eq. (8).

First, the infinitesimal gauge transformation of $h_{M N}$ due to a vector field $\xi^{M}$ is expressed as

$$
h_{M N} \rightarrow h_{M N}-\xi_{M ; N}-\xi_{N ; M},
$$

where $\xi_{M} \equiv g_{M N}^{(0)} \xi^{N}$, and ";" denotes the covariant derivative compatible with the background metric $\bar{g}_{M N}^{(0)}$.

Next, we expand $h_{M N}$ and $\xi_{M}$ by harmonics on $S^{d}$, which are defined in Appendix B, as

$$
\begin{aligned}
h_{M N} d x^{M} d x^{N}= & \sum_{l m}\left[h_{\mu \nu}^{l m} Y_{l m} d x^{\mu} d x^{\nu}+2\left\{h_{(T) \mu}^{l m}\left(V_{(T) l m}\right)_{i}+h_{(L) \mu}^{l m}\left(V_{(L) l m}\right)_{i}\right\} d x^{\mu} d x^{i}\right. \\
& \left.+\left\{h_{(T)}^{l m}\left(T_{(T) l m}\right)_{i j}+h_{(L T)}^{l m}\left(T_{(L T) l m}\right)_{i j}+h_{(L L)}^{l m}\left(T_{(L L) l m}\right)_{i j}+h_{(Y)}^{l m}\left(T_{(Y) l m}\right)_{i j}\right\} d x^{i} d x^{j}\right],
\end{aligned}
$$

and 


$$
\xi_{M} d x^{M}=\sum_{l m}\left[\xi_{\mu}^{l m} Y_{l m} d x^{\mu}+\left\{\xi_{(T)}^{l m}\left(V_{(T) l m}\right)_{i}+\xi_{(L)}^{l m}\left(V_{(L) l m}\right)_{i}\right\} d x^{i}\right]
$$

Here, the coefficients $h_{\mu \nu}^{l m}, h_{(T) \mu}^{l m}, h_{(L) \mu}^{l m}, h_{(T)}^{l m}, h_{(L T)}^{l m}, h_{(L L)}^{l m}, h_{(Y)}^{l m}, \xi_{\mu}^{l m}, \xi_{(T)}^{l m}$ and $\xi_{(L)}^{l m}$ depend only on the four-dimensional coordinates $x^{\mu}$, while the harmonics depend only on the coordinates $x^{i}$ on $S^{d}$.

Hence, by substituting the above expansions into Eq. (C1), we obtain the infinitesimal gauge transformation of each coefficient.

$$
\begin{aligned}
h_{\mu \nu}^{l m} & \rightarrow h_{\mu \nu}^{l m}-\left(\frac{b}{b_{0}}\right)^{-d}\left\{\nabla_{\mu}\left[\left(\frac{b}{b_{0}}\right)^{d / 2} \xi_{\nu}^{l m}\right]+\nabla_{\nu}\left[\left(\frac{b}{b_{0}}\right)^{d / 2} \xi_{\mu}^{l m}\right]\right\}+d\left(\frac{b}{b_{0}}\right)^{-d / 2} g_{\mu \nu} g^{\alpha \beta} \xi_{\alpha}^{l m} \partial_{\beta} \ln \left(\frac{b}{b_{0}}\right), \\
h_{(T) \mu}^{l m} & \rightarrow h_{(T) \mu}^{l m}-b^{2} \partial_{\mu}\left[b^{-1} \xi_{(T)}^{l m}\right], \\
h_{(L) \mu}^{l m} & \rightarrow h_{(L) \mu}^{l m}-\left(\frac{b}{b_{0}}\right)^{-d / 2} \xi_{\mu}^{l m}-b^{2} \partial_{\mu}\left[b^{-1} \xi_{(L)}^{l m}\right], \\
h_{(T)}^{l m} & \rightarrow h_{(T)}^{l m}, \\
h_{(L T)}^{l m} & \rightarrow h_{(L T)}^{l m}-b \xi_{(T)}^{l m}, \\
h_{(L L)}^{l m} & \rightarrow h_{(L L)}^{l m}-b \xi_{(L)}^{l m}, \\
h_{(Y)}^{l m} & \rightarrow h_{(Y)}^{l m}-2 b^{2}\left(\frac{b}{b_{0}}\right)^{d / 2} g^{\mu \nu} \xi_{\mu}^{l m} \partial_{\nu} \ln \left(\frac{b}{b_{0}}\right)+\frac{2 l(l+d-1)}{d} b \xi_{(L)}^{l m},
\end{aligned}
$$

where $\nabla$ is the covariant derivative compatible with the 4-dimensional metric $g_{\mu \nu}$.

Therefore, by choosing $\xi_{\mu}^{l m}, \xi_{(T)}^{l m}$ and $\xi_{(L)}^{l m}$ as

$$
\begin{aligned}
& \xi_{(T)}^{l m}=b^{-1} h_{(L T)}^{l m} \quad(l \geq 2), \\
& \xi_{(L)}^{l m}=b^{-1} h_{(L L)}^{l m} \quad(l \geq 2), \\
& \xi_{\mu}^{l m}=\left(\frac{b}{b_{0}}\right)^{d / 2} h_{(L) \mu}^{l m}-b^{2}\left(\frac{b}{b_{0}}\right)^{d / 2} \partial_{\mu}\left[b^{-1} \xi_{(L)}^{l m}\right] \quad(l \geq 1), \\
& \xi_{(L)}^{1 m}=-\frac{1}{2 b} h_{(Y)}^{1 m}+b\left(\frac{b}{b_{0}}\right)^{d / 2} g^{\mu \nu} \xi_{\mu}^{1 m} \partial_{\nu} \ln \left(\frac{b}{b_{0}}\right),
\end{aligned}
$$

we can always make a gauge transformation such that

$$
\begin{aligned}
& h_{(L T)}^{l m} \rightarrow 0 \quad(l \geq 2), \\
& h_{(L L)}^{l m} \rightarrow 0 \quad(l \geq 2), \\
& h_{(L) \mu}^{l m} \rightarrow 0 \quad(l \geq 1), \\
& h_{(Y)}^{1 m} \rightarrow 0 \text {. }
\end{aligned}
$$

Moreover, $h_{\mu \nu}^{00}$ and $h_{(Y)}^{00}$ can be eliminated by redefining the 4-dimensional metric $g_{\mu \nu}$ (or $\hat{g}_{\mu \nu}$ ) and 4-dimensional scalar field $b$ (or $\sigma)$. It can be easily shown that the gauge transformation of $h_{\mu \nu}^{00}$ and $h_{(Y)}^{00}$ is equivalent to the 4-dimensional gauge transformation.

Therefore, we obtain the following expansion of $h_{M N}$ :

$$
h_{M N} d x^{M} d x^{N}=\sum_{l m}\left[h_{\mu \nu}^{l m} Y_{l m} d x^{\mu} d x^{\nu}+2 h_{(T) \mu}^{l m}\left(V_{(T) l m}\right)_{i} d x^{\mu} d x^{i}+\left\{h_{(T)}^{l m}\left(T_{(T) l m}\right)_{i j}+h_{(Y)}^{l m}\left(T_{(Y) l m}\right)_{i j}\right\} d x^{i} d x^{j}\right],
$$

where the summations are taken over $l \geq 1$ for the scalar and vector harmonics, and over $l \geq 2$ for the tensor harmonics.

The remaining gauge freedom is given by $\xi_{(T)}^{1 m}$ and $\xi_{\mu}^{00}$. The first one generates the gauge transformation of $h_{(T) \mu}^{l m}$ as

$$
\left(h_{(T)}^{1 m}\right)_{\mu} \rightarrow\left(h_{(T)}^{1 m}\right)_{\mu}-b^{2} \partial_{\mu}\left[b^{-1} \xi_{(T)}^{1 m}\right] .
$$


Another remaining gauge freedom $\xi_{\mu}^{00}$ generates the usual 4-dimensional gauge transformation.

[1] M. B. Green, J. H. Schwartz, E. Witten, Superstring theory (Cambridge University Press, 1987); J. Polchinski, String Theory IE II (Cambridge University Press, Cambridge, England 1998).

[2] J. H. Schwartz, Nucl. Phys. Suppl. B55, 1 (1997).

[3] T. Applquist, A. Chodos and P. G. O. Freund, Modern Kaluza-Klein theories, (Benjamin / Cummings, 1985).

[4] L. Randall and R. Sundrum, hep-th/9906064.

[5] E. W. Kolb and R. Slansky, Phys. Lett. B135, 378 (1984).

[6] N. Arkani-Hamed, S. Dimopoulos, N. Kaloper and J. March-Russell, hep-ph/9903224, 9903239; James M. Cline, hepph/9904495; E. Halyo, hep-ph9907223; E. E. Flanagan, S.-H. H. Tye and I. Wasserman, hep-ph/99099373; L. Mersini, hep-ph/9909494.

[7] P. Candelas and S. Weinberg, Nucl. Phys. B237, 397(1984)

[8] L. Landau and E. Lifschitz, Mechanics (Pergamon, Oxford, 1960).

[9] For example, L. Kofman, A. Linde and A. A. Starobinsky, Phys. Rev. D56, 3258 (1997), and references therein.

[10] S. Mukohyama, Phys. Rev. D157, 6191 (1998)

[11] For example, K. Maeda, Phys. Lett. B186, 33 (1987); L. Amendola, E. W. Kolb, M. Litterio and F. Occhionero, Phys. Rev. D42, 1944 (1990).

[12] P. G. O. Freund and M. A. Rubin, Phys. Lett. 97B, 233 (1980).

[13] W. D. Goldberger and M. B. Wise, hep-ph/9907447; W. D. Goldberger and M. B. Wise, hep-ph/9911457, C. Csaki, M. Graesser, L. Randal and J. Terning, hep-ph/9911406; P. Kanti, I. I. Kogan, K. A. Olive and M. Pospelov, hep-ph/9912266.

[14] M. A. Rubin and C. R. ordóñez, J. Math. Phys. 25, 2888 (1984); 26, 65 (1985). 